Troupin, D., R. Nathan and G. G. Vendramin (2006): Analysis of spatial genetic structure in an expanding Pinus halepensis population reveals development of fine-scale genetic clustering over time. Mol. Ecol. 15: 3617-3630.

Valbuena-Carabaña, M., S. C. GonzÁlez-Martínez, O. J. HARDY and L. GIL (2007): Fine-scale spatial genetic structure in mixed oak stands with different levels of hybridization. Mol. Ecol. 16: 1207-1219.

VeKEMAns, X. and O. J. HARDY (2004): New insights from fine-scale spatial genetic structure analyses in plant populations. Mol. Ecol. 13: 921-935.

Vos, P., R. Hogers and M. Bleeker (1995): AFLP: a new concept for DNA fingerprinting. Nucleic Acids Res. 23: 4407-4414.
Wehenkel, C., J. H. Martínez-Guerrero, A. PinedoAlvAREZ and A. CARRILlO (2012): Adaptive genetic differentiation in Picea chihuahuana M. caused by different copper concentrations in the top soil. Forstarchiv 83: 48-51.

WeHenkel, C. and C. SÁenz-Romero (2012): Estimating genetic erosion using the example of Picea chihuahuana Martínez. Tree Genet. Genomes 8: 1085-1094.

Wehenkel, C., C. SÁenz-Romero and J. P. JaramilloCORREA (2014): Estimating genetic erosion in threatened conifers: the example of Picea chihuahuana Martínez, Chapter 15: 20 pages in Ahuja, M. R. and Jain, S.M. (eds.): Genetic Erosion and Biodiversity, Springer SBM, The Netherlands, in press.

WRIGHT, S. (1943): Isolation by distance. Genetics 28 . $114-138$

\title{
Inference of allelic dosages and inheritance modes in tetraploids: a case study in Betula apoiensis with a putative hybrid origin
}

\author{
By T. NAGAmitsu ${ }^{1), *)}$, T. KaWAHARA ${ }^{1)}$ and A. KAnAZASHI ${ }^{2)}$
}

(Received 23 ${ }^{\text {rd }}$ April 2014)

\begin{abstract}
In tetraploids, inference of allelic dosages and inheritance modes is difficult due to the ambiguous number of allele copies and the variation between disomic and tetrasomic patterns. Considering the biases of amplification and the overlaps of stutter products in polymerase chain reactions, we inferred tetraploid genotypes at three nuclear microsatellite loci in progeny arrays from six reciprocal crosses among three parents of Betula

\footnotetext{
1) TERUYOSh NAgAmitsu and TAKAYUKi Kawahara: Forestry and Forest Products Research Institute (FFPRI), Tsukuba 3058687, Japan.

2) AyaKo KanazAshi: Hokkaido Research Center, Forestry and Forest Products Research Institute (FFPRI), Sapporo 062 8516, Japan.

*) Corresponding author: Dr. TeRuYoshi NAgAmitsu. Department of Forest Genetics, Forestry and Forest Products Research Institute (FFPRI), Tsukuba 305-8687, Japan.

Phone: +81-29-829-8262; Fax: +81-29-874-3720

E-mail: nagamit@ffpri.affrc.go.jp
}

apoiensis with a putative hybrid origin. In each cross, we assigned diploid genotypes to gametes on the basis of the tetraploid genotypes of the parents and their progeny and observed the frequencies of the gamete genotypes. Segregation patterns of the observed gamete genotypes indicated few null alleles in the progeny arrays and tetrasomic inheritance with rare double reduction. This mode of inheritance was consistent between genders and between mates in the crosses. This result suggests that our method to infer tetraploid genotypes in nuclear microsatellites is successful in family samples with few null alleles when the amplification biases and the stutter-product overlaps are accessed properly.

Key words: Allelic configuration; Microsatellite; Polyploidy; Reciprocal cross; Segregation.

\section{Introduction}

Polyploidy is an important aspect in evolution and speciation in plants (SOLTIS et al., 2003; DUFRESNE et al., 2014). High levels of heterozygosity due to the duplica- 
tion of genomes may provide a genetic buffer to prevent inbreeding depression and genetic drift (SoLTIS and Soltis, 2000). Combination and rearrangement of genomes from different progenitors may promote adaptation to environments intermediate between habitats of the progenitors or those that they have not experienced (LEITCH and BENNETT, 1997). Allopolyploids, which comprise genomes of different species, are expected to show fixed heterozygosity and disomic inheritance due to the formation of bivalents at meiosis. On the other hand, autopolyploids, which consist of genomes of the same species, are presumed to exhibit polysomic inheritance owing to the formation of multivalents (RONFORT et al., 1998). In polysomic inheritance, various arrangements of chromosomal paring result in diversity between random chromosome segregation and random chromatid segregation (BEVER and FELBER, 1992). In the former, two sister chromatids of a chromosome never sort into the same gamete. In the latter, the two sister chromatids migrate into the same gamete, which is called double reduction, increasing the frequency of homozygous gametes.

In polyploids, genotyping has been difficult due to the ambiguous number of copies in each allele (DE SiLva et al., 2005). The amount of polymerase chain reaction (PCR) products was used to infer the allelic dosage at nuclear microsatellite loci in polyploid plants using the MAC-PR (microsatellite DNA allele counting-peak ratios) method (EssELINK et al., 2004; NYBOM et al., 2004; Lo et al., 2009). In PCR amplification of microsatellites, unequal amplification of alleles with different fragment lengths, replication slippage result- ing in stutter products, and null alleles relatively frequent in polyploids are main difficulties in the inference of polyploid genotypes (DUFRESNE et al., 2014). LANDERGOTT et al. (2006) and LUTTIKHUIZEN et al. (2007) took the amplification biases and the stutter-product overlaps into their inference of allelic dosages although their quantitative methods were unclear. Family samples are useful to detect null alleles because the inheritance of null alleles of parents results in unexpected genotypes in their progeny (DAKIN and AvISE, 2004). Here, we inferred tetraploid genotypes in progeny arrays using a correction of the peak heights of PCR products, from which we excluded the amplification biases among alleles and the overlaps of stutter peaks with a target peak of each allele.

Polyploidy is common in birches (the genus Betula, Betulaceae), which are trees and shrubs in arctic, boreal, and temperate climate zones of the northern hemisphere. In Betula, the basic chromosome number is thought to be $n=14$, and thus, the diploid chromosome number is $2 n=28$ (ERIKKSON and JONSSON, 1986). There are natural polyploids with chromosome numbers such as $2 n=56,70,84,112$, and 140 (FuRLOW, 1990). Another basic chromosome number is suggested to be $n=7$, according to the presence of multivalents in diploid $(2 n=28)$ trees and the fairly regular division of chromosomes in triploid $(2 n=42)$ trees (BROWN and ALDAWOoDy, 1979). Phylogenetic reconstructions of birches indicate that several polyploidization events occurred independently (JÄRVINEN et al., 2004; LI et al., 2005; SCHENK et al., 2008). Hybridization and introgression are common between diploids and between tetraploids

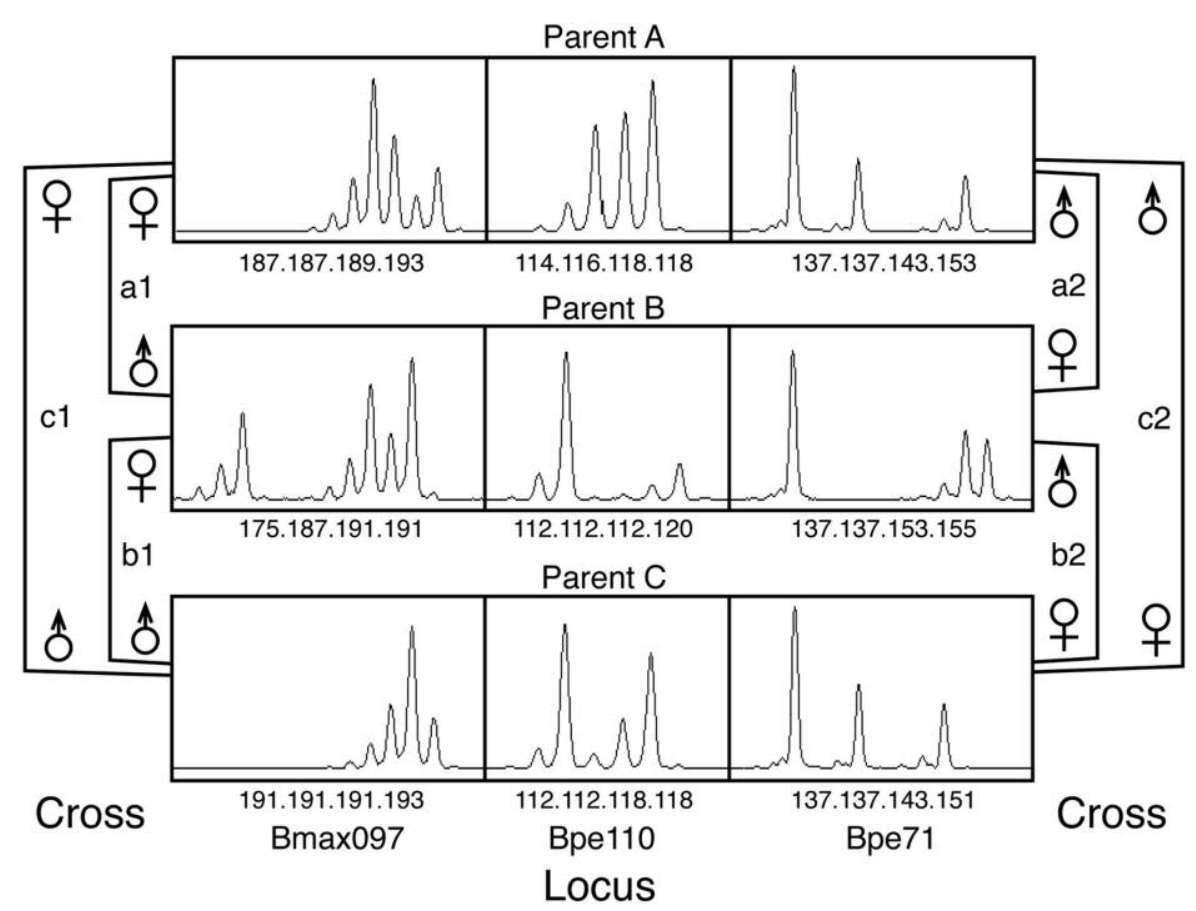

Figure 1. - Electropherograms and tetraploid genotypes at three loci of nuclear microsatellites in three parents of Betula apoiensis in six reciprocal crosses. 
(PALME et al., 2004) as well as between diploids and tetraploids through triploids (THÓRSSON et al., 2001) in birches. Betula apoiensis is a dwarf birch species endemic to a mountain in Hokkaido, Japan (NAGAMITSU et al., 2006a). This tetraploid species is thought to have originated from hybridization between two tetraploid progenitor species, $B$. ermanii and $B$. ovalifolia (NAGAMITSU et al., 2006b) although the speciation process of B. apoiensis is unclear (SCHUMER et al., 2014). The knowledge of its inheritance mode may help to understand the evolutionary history of $B$. apoiensis.

In order to examine the inheritance mode in B.apoiensis, we conducted reciprocal crosses and inferred tetraploid genotypes of parents and progeny in the crosses. Next, we assigned diploid genotypes to gametes using the tetraploid genotypes of the parents and progeny. Finally, on the basis of segregation patterns in the gamete genotypes, we evaluated the inference of tetraploid genotypes and discussed the inheritance modes in birches.

\section{Materials and methods}

\section{Reciprocal crosses}

Betula apoiensis Nakai is distributed only on Mt. Apoi, Hokkaido, Japan $\left(42^{\circ} 6.5^{\prime} \mathrm{N}, 143^{\circ} 1.2^{\prime} \mathrm{E}, \quad 580-810 \mathrm{~m}\right.$ altitude) (NAGAMITSU et al., 2006a, b). In 2003, we conducted six reciprocal crosses (a1, a2, b1, b2, c1, and c2) among three parent trees (A, B, and C; Fig. 1) growing at altitudes from 600 to $640 \mathrm{~m}$ on Mt. Apoi. There were three pairs of parents, and each parent was crossed with the other two parents as a mother and as a father (Fig. 1).

Before flowers opened, we removed all male inflorescences on two branches with female inflorescences of each parent tree. On May $8^{\text {th }}$, we enclosed these branches with paper bags for pollination $(20$ x $40 \mathrm{~cm}$, Japan Forest Tree Breeding Association, Tokyo). We collected mature male inflorescences from other branches of the parent trees on May $20^{\text {th }}$ and stored them in paper envelopes at $4^{\circ} \mathrm{C}$. We dried and warmed the stored male inflorescences to open the anthers a day before the following cross treatment. In the morning of May $21^{\text {st }}$ and $28^{\text {th }}$, we extracted pollen from the anthers of a designated father and injected it into a paper bag enclosing a branch of a designated mother using a pollen gun (Japan Forest Tree Breeding Association). After the flowering ended, we removed the paper bags. On September $8^{\text {th }}$ and $9^{\text {th }}$, we collected all of the fruits on those branches of the parent trees.

We sowed seeds of the collected fruit on a moistened filter paper and a 2-mm-thick sponge disc in a 9-cmdiameter Petri dish. We stored the seeds in the Petri dishes at $4{ }^{\circ} \mathrm{C}$ in darkness for one week and then incubated them at $20^{\circ} \mathrm{C}$ in a $14: 10$-hour day-night cycle. We collected seeds that had germinated (seedlings) from the Petri dishes after the incubation for two weeks.

\section{DNA extraction and PCR amplification}

We extracted total DNA from 48 seedlings generated from each cross using the PrepMan Ultra (Applied
Biosystems, Foster City) and from leaves collected from the three parent trees using the DNeasy Plant Mini Kit (Qiagen, Hilden).

We designed primer pairs for three nuclear microsatellite loci characterized in B. pendula (KULJU et al., 2004): Bpe71 (AF310854), Bpe110 (AF310856), and Bpe54 (AF310862). Sequences of the primer pair for Bpe71 were 5'-GTTTTGGGTTTCCACTTCCA-3' and 5'ACTGGTAATACCTTTACCAAGCC-3', those for Bpe110 were 5'-CAGCCTGTGTCAAACATAACA-3' and 5'-GCACAACCACTGCTCCTTT-3', and those for Bpe54 were 5'ACCTGCAGATTAGAAAGCAT-3' and 5'-GAAGCTGTCGAAAATGTTCA-3'. Additional primer pairs for two loci, Bmax097 and Bmax624, which were isolated from B. maximowicziana Regel (OGYU et al., 2003), could be applied to B. apoiensis. Among the five loci, PCR amplification was successful at three loci, Bmax097, Bpe110, and Bpe71, which we examined in the following analysis.

Table 1. - The relative amplification rate for a target peak among alleles and the relative height of stutter peaks against a target peak of each allele, which were used to correct the height of the target peak, at three loci of nuclear microsatellites in Betula apoiensis.

\begin{tabular}{rrrr}
\hline \multirow{2}{*}{$\begin{array}{c}\text { Locus } \\
\text { Allele }\end{array}$} & \multicolumn{2}{c}{\begin{tabular}{c} 
Relative ampli- \\
\cline { 3 - 4 } fication rate
\end{tabular}} & Felative height of stutter peaks \\
\hline Bmax097 & & & Second \\
175 & 1.8 & 0.3 & 0.1 \\
187 & 1.0 & 0.4 & 0.2 \\
189 & 1.0 & 0.4 & 0.2 \\
191 & 0.9 & 0.5 & 0.2 \\
193 & 0.9 & 0.5 & 0.2 \\
\hline
\end{tabular}

Bpe110

\begin{tabular}{llll}
112 & 1.0 & 0.2 & 0.0 \\
114 & 0.8 & 0.3 & 0.1 \\
116 & 0.7 & 0.4 & 0.1 \\
118 & 0.7 & 0.4 & 0.1 \\
120 & 0.7 & 0.4 & 0.2 \\
\hline
\end{tabular}

Bpe71

\begin{tabular}{llll}
137 & 1.0 & 0.1 & 0.0 \\
143 & 0.8 & 0.1 & 0.0 \\
151 & 0.7 & 0.2 & 0.1 \\
153 & 0.7 & 0.3 & 0.1 \\
155 & 0.6 & 0.3 & 0.1 \\
\hline
\end{tabular}


Table 2. - The observed frequencies of gamete genotypes that created progeny from three parents of Betula apoiensis in six reciprocal crosses at three loci of nuclear microsatellites.

\begin{tabular}{|c|c|c|c|c|c|c|c|c|}
\hline \multicolumn{9}{|l|}{ Bmax097 } \\
\hline \multirow{2}{*}{$\begin{array}{l}\text { Cross a1 } \\
\text { Mother A }\end{array}$} & \multirow{2}{*}{$\begin{array}{l}\text { Father B } \\
\text { Gamete }\end{array}$} & \multicolumn{6}{|c|}{175.187 .191 .191} & \multirow[b]{2}{*}{ Total } \\
\hline & & 175.175 & 175.187 & 175.191 & 187.187 & 187.191 & 191.191 & \\
\hline \multirow[t]{7}{*}{187.187 .189 .193} & 187.187 & & & & & 3 & 2 & 5 \\
\hline & 187.189 & & 1 & 5 & & 10 & 1 & 17 \\
\hline & 187.193 & & 2 & 3 & & 4 & 4 & 13 \\
\hline & 189.189 & & & & & & & 0 \\
\hline & 189.193 & & & 1 & & 2 & 3 & 6 \\
\hline & 193.193 & & & & & & & 0 \\
\hline & Total & 0 & 3 & 9 & 0 & 19 & 10 & 41 \\
\hline \multirow{2}{*}{$\begin{array}{l}\text { Cross a2 } \\
\text { Father A }\end{array}$} & Mother B & 175.187 .19 & .191 & & & & & \\
\hline & Gamete & 175.175 & 175.187 & 175.191 & 187.187 & 187.191 & 191.191 & Total \\
\hline \multirow[t]{7}{*}{187.187 .189 .193} & 187.187 & 2 & & 1 & & 3 & & 6 \\
\hline & 187.189 & & & 2 & & 4 & & 6 \\
\hline & 187.193 & & & 1 & & 3 & 2 & 6 \\
\hline & 189.189 & & & & & & & 0 \\
\hline & 189.193 & & & & & 2 & & 2 \\
\hline & 193.193 & & & & & & & 0 \\
\hline & Total & 2 & 0 & 4 & 0 & 12 & 2 & 20 \\
\hline
\end{tabular}

Other offspring: $175.175 .175 .175=1,175.189 .191 .191=1,191.191 .191 .193=1$

\begin{tabular}{lcrrrr}
\hline Cross b1 & Father C & 191.191 .191 .193 & & \\
\cline { 3 - 5 } Mother B & Gamete & 191.191 & 191.193 & 193.193 & Total \\
\hline 175.187 .191 .191 & 175.175 & & & & 0 \\
& 175.187 & 6 & 1 & & 7 \\
& 175.191 & 13 & 2 & & 15 \\
& 187.187 & & & & 0 \\
& 187.191 & 5 & 3 & & 8 \\
& 191.191 & 1 & 2 & & 3 \\
\hline Total & 25 & 8 & 0 & 33 \\
\hline
\end{tabular}

\begin{tabular}{|c|c|c|c|c|c|}
\hline \multirow{3}{*}{$\begin{array}{l}\text { Cross b2 } \\
\text { Father B } \\
175.187 .191 .191\end{array}$} & \multicolumn{4}{|c|}{ Mother C 191.191.191.193 } & \multirow[b]{2}{*}{ Total } \\
\hline & Gamete & 191.191 & 191.193 & 193.193 & \\
\hline & 175.175 & & & & 0 \\
\hline & 175.187 & 8 & 2 & & 10 \\
\hline & 175.191 & 6 & 4 & & 10 \\
\hline & 187.187 & & & & 0 \\
\hline & 187.191 & 10 & 8 & & 18 \\
\hline & 191.191 & 2 & 5 & & 7 \\
\hline & Total & 26 & 19 & 0 & 45 \\
\hline$\overline{\text { Cross c1 }}$ & Father C & 191.191 .19 & .193 & & \\
\hline Mother A & Gamete & 191.191 & 191.193 & 193.193 & Total \\
\hline$\overline{187.187 .189 .193}$ & 187.187 & 2 & 5 & & 7 \\
\hline & 187.189 & 8 & 9 & & 17 \\
\hline & 187.193 & 6 & 6 & & 12 \\
\hline & 189.189 & & & & 0 \\
\hline & 189.193 & 6 & 4 & & 10 \\
\hline & 193.193 & & & & 0 \\
\hline & Total & 22 & 24 & 0 & 46 \\
\hline$\overline{\text { Cross c2 }}$ & Mother C & 191.191 .19 & .193 & & \\
\hline Father A & Gamete & 191.191 & 191.193 & 193.193 & Total \\
\hline 187.187 .189 .193 & 187.187 & 3 & 1 & & $\overline{4}$ \\
\hline & 187.189 & 12 & 10 & & 22 \\
\hline & 187.193 & 3 & 7 & & 10 \\
\hline & 189.189 & & & & 0 \\
\hline & 189.193 & 4 & 6 & & 10 \\
\hline & 193.193 & & & & 0 \\
\hline & Total & 22 & 24 & 0 & 46 \\
\hline
\end{tabular}

We carried out PCRs in $15 \mu \mathrm{L}$ of a mixture containing $2.5 \mathrm{mM}$ of $\mathrm{MgCl}_{2}$, a 0.5 unit of AmpliTaq Gold DNA Polymerase in a GeneAmp PCR Gold Buffer (Applied Biosystems), $0.4 \mu \mathrm{M}$ of each primer, $0.2 \mathrm{mM}$ of each dNTP, and $1 \mu \mathrm{L}$ of the template DNA solution using a GeneAmp 9700 thermal cycler (Applied Biosystems) pro- grammed for 9 min at $95^{\circ} \mathrm{C}$, followed by 28 cycles of $30 \mathrm{~s}$ at $95^{\circ} \mathrm{C}, 30 \mathrm{~s}$ at $56^{\circ} \mathrm{C}$, and $45 \mathrm{~s}$ at $72^{\circ} \mathrm{C}$. We measured the fragment length and peak height of the PCR products using an ABI PRISM 3100-Avant Genetic Analyzer with POP4 in 36-cm capillaries and GeneScan Analysis Software (Applied Biosystems). 
Table 2. - (Continued).

\begin{tabular}{|c|c|c|c|c|c|}
\hline \multicolumn{6}{|c|}{ Bpe110 } \\
\hline \multirow{2}{*}{$\begin{array}{l}\text { Cross al } \\
\text { Mother A }\end{array}$} & \multicolumn{4}{|c|}{$\begin{array}{ll}\text { Father B } & 112.112 .112 .120\end{array}$} & \multirow[b]{2}{*}{ Tota } \\
\hline & Gamete & 112.112 & 112.120 & 120.120 & \\
\hline \multirow[t]{7}{*}{114.116 .118 .118} & 114.114 & & & & 0 \\
\hline & 114.116 & 2 & 3 & & 5 \\
\hline & 114.118 & 9 & 7 & & 16 \\
\hline & 116.116 & & & & 0 \\
\hline & 116.118 & 5 & 12 & & 17 \\
\hline & 118.118 & 3 & 4 & & 7 \\
\hline & Total & 19 & 26 & 0 & 45 \\
\hline$\overline{\text { Cross a2 }}$ & \multicolumn{4}{|c|}{ Mother B 112.112 .112 .120} & \\
\hline Father A & Gamete & 112.112 & 112.120 & 120.120 & Total \\
\hline \multirow[t]{7}{*}{114.116 .118 .118} & 114.114 & & & & 0 \\
\hline & 114.116 & 7 & 2 & & 9 \\
\hline & 114.118 & & 7 & & 7 \\
\hline & 116.116 & & & & 0 \\
\hline & 116.118 & 5 & 6 & & 11 \\
\hline & 118.118 & 2 & 2 & & 4 \\
\hline & Total & 14 & 17 & 0 & 31 \\
\hline$\overline{\text { Cross b1 }}$ & \multicolumn{4}{|c|}{ 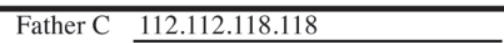 } & \\
\hline Mother B & Gamete & 112.112 & 112.118 & 118.118 & Total \\
\hline \multirow[t]{4}{*}{112.112 .112 .120} & 112.112 & 6 & 15 & & 21 \\
\hline & 112.120 & 2 & 9 & 4 & 15 \\
\hline & 120.120 & & & & 0 \\
\hline & Total & 8 & 24 & 4 & 36 \\
\hline$\overline{\text { Cross b2 }}$ & \multicolumn{4}{|c|}{ Mother C 112.112 .118 .118} & \\
\hline Father B & Gamete & 112.112 & 112.118 & 118.118 & Total \\
\hline \multirow[t]{4}{*}{112.112 .112 .120} & 112.112 & 2 & 17 & 4 & 23 \\
\hline & 112.120 & & 13 & 3 & 16 \\
\hline & 120.120 & & & & 0 \\
\hline & Total & 2 & 30 & 7 & 39 \\
\hline$\overline{\text { Cross } \mathrm{cl}}$ & \multicolumn{4}{|c|}{$\begin{array}{ll}\text { Father C } & 112.112 .118 .118\end{array}$} & \\
\hline Mother A & Gamete & 112.112 & 112.118 & 118.118 & Total \\
\hline \multirow{7}{*}{114.116 .118 .118} & 114.114 & & & & 0 \\
\hline & 114.116 & 1 & 7 & & 8 \\
\hline & 114.118 & 3 & 9 & & 12 \\
\hline & 116.116 & & & & 0 \\
\hline & 116.118 & 1 & 11 & 3 & 15 \\
\hline & 118.118 & & 6 & 2 & 8 \\
\hline & Total & 5 & 33 & 5 & 43 \\
\hline$\overline{\text { Cross c2 }}$ & \multicolumn{4}{|c|}{ Mother C 112.112 .118 .118} & \\
\hline Father A & Gamete & 112.112 & 112.118 & 118.118 & Total \\
\hline \multirow[t]{7}{*}{114.116 .118 .118} & 114.114 & & & & 0 \\
\hline & 114.116 & & 8 & 2 & 10 \\
\hline & 114.118 & 1 & 5 & 2 & 8 \\
\hline & 116.116 & & & & 0 \\
\hline & 116.118 & & 11 & 4 & 15 \\
\hline & 118.118 & 3 & 8 & 3 & 14 \\
\hline & Total & 4 & 32 & 11 & 47 \\
\hline
\end{tabular}

\section{Allelic dosages}

We inferred tetraploid genotypes of the three parents and their progeny at the three loci according to the observed peak height, $h$. The PCR products of nuclear microsatellites usually show different amplification rates among alleles depending on the fragment length and often have some stutter peaks one or two sequence repeats shorter than a target peak of each allele (Fig. 1). To correct the height of the target peak, we used three parameters: the relative amplification rate, $r$, for a target peak among alleles and the relative heights of the first and second stutter peaks, $f$ and $s$, respectively, against a target peak of each allele (Table 1). First, using electropherograms that we discriminated target and stutter peaks of each allele from peaks of the other alleles, we estimated the parameters, $f_{i}$ and $s_{i}$, for each allele with the fragment length, $i$ (bp). Second, using electropherograms that we recognized target peaks of four alleles simultaneously, we estimated the relative amplification rate, $r_{i}$, among the alleles, as setting $r$ of a certain allele to 1 . Finally, we calculated the corrected peak height, $g_{i}$, from the observed peak height, $h_{i}$, and the three parameters, $f_{i}, s_{i}$, and $r_{i}$, for each allele with the fragment length, $i$ (bp), in microsatellites with dinucleotide repeat motifs, as follows:

$$
g_{i}=r_{i}\left(h_{i}-f_{i} g_{i+2}-s_{i} g_{i+4}\right) \text {. }
$$

The ratios of the corrected peak heights for observed alleles in each tetraploid individual should be $0.25,0.5$, 0.75 , and 1 , which correspond to the allele copy numbers, $1,2,3$, and 4 , respectively. On the basis of a frequency distribution of the peak height ratio in the parents and their progeny, we inferred the allelic dosages at each locus from the calculated ratios.

\section{Inheritance modes}

We assigned diploid genotypes at each locus to gametes that derived from the tetraploid parents in each cross by matching the inferred genotypes between the parents and their progeny (Table 2). We compared the observed frequencies of the gamete genotypes among the reciprocal crosses of each parent using a Chisquared test (chisq.test in $\mathrm{R}$ 3.0.2) ( $\mathrm{R}$ DEVELOPMENT CORE TEAM, 2013) to verify whether the segregation patterns of the gamete genotypes were consistent between genders and between mates. For example, we compared the observed frequencies of the gamete genotypes from parent A among four crosses, a1 as the mother and a2 as the father mated with parent B as well as c1 as the mother and c2 as the father mated with parent $\mathrm{C}$ (Fig. 1). When the segregation patterns were consistent among the four crosses, we pooled the observed frequencies of gamete genotypes at each locus from each parent among the crosses.

We compared the observed frequencies of gamete genotypes with the frequencies expected from tetrasomic inheritance without double reduction (1) and with double reduction at the maximum incidence (2) and some segregation patterns in disomic inheritance (3). In case (1), six possible combinations of four alleles in each parent occur at the same probability, 1/6, in gamete genotypes (BEVER and FELBER, 1992). When double reduction occurs at the probability, $\alpha / 2$, half of the index of separation (MATHER, 1936), two sister chromatids of a chromosome migrate into the same gamete, resulting in a homozygous gamete. Thus, the homozygous gamete genotype with each of four alleles occurs at the probability, $\alpha / 8$, and the other genotypes with any combinations of these alleles occur at the probability, $(2-\alpha) / 12$ (BEVER and FELBER, 1992). Because the maximum frequency of double reduction is thought to be 1/7 (BEVER and FELBER, 1992), we applied $\alpha=2 / 7$ to case (2). In disomic inheritance, there are complete fidelity in the pairing of homologous chromosomes and complete rejection of the 
Table 2. - (Continued).

\begin{tabular}{|c|c|c|c|c|c|c|c|c|}
\hline \multicolumn{9}{|l|}{ Bpe71 } \\
\hline \multirow{2}{*}{$\begin{array}{l}\text { Cross b1 } \\
\text { Mother B }\end{array}$} & \multicolumn{7}{|c|}{ Father C $\quad 137.137 .143 .151$} & \multirow[b]{2}{*}{ Total } \\
\hline & Gamete & 137.137 & 137.143 & 137.151 & 143.143 & 143.151 & 151.151 & \\
\hline \multirow[t]{7}{*}{137.137 .153 .155} & 137.137 & 1 & 4 & 3 & & 1 & & $\overline{9}$ \\
\hline & 137.153 & 3 & 5 & 2 & & 4 & & 14 \\
\hline & 137.155 & 2 & 5 & 6 & & 1 & & 14 \\
\hline & 153.153 & & & & & & & 0 \\
\hline & 153.155 & 2 & 2 & 1 & & & & 5 \\
\hline & 155.155 & & & & & & & 0 \\
\hline & Total & 8 & 16 & 12 & 0 & 6 & 0 & 42 \\
\hline$\overline{\text { Cross b2 }}$ & \multicolumn{7}{|c|}{ Mother C 137.137 .143 .151} & \\
\hline Father B & Gamete & 137.137 & 137.143 & 137.151 & 143.143 & 143.151 & 151.151 & Total \\
\hline \multirow[t]{7}{*}{137.137 .153 .155} & 137.137 & & 3 & 3 & & 2 & & 8 \\
\hline & 137.153 & 1 & 6 & 5 & & 1 & & 13 \\
\hline & 137.155 & 1 & 6 & 8 & & 3 & & 18 \\
\hline & 153.153 & & & & & & & 0 \\
\hline & 153.155 & & 1 & 1 & & 4 & & 6 \\
\hline & 155.155 & & & & & & & 0 \\
\hline & Total & 2 & 16 & 17 & 0 & 10 & 0 & 45 \\
\hline
\end{tabular}

pairing of homoeologous chromosomes, which differentiate two alleles from the others among four alleles in each parent (Olson, 1997). Because there are usually multiple groupings of these alleles, we examined each
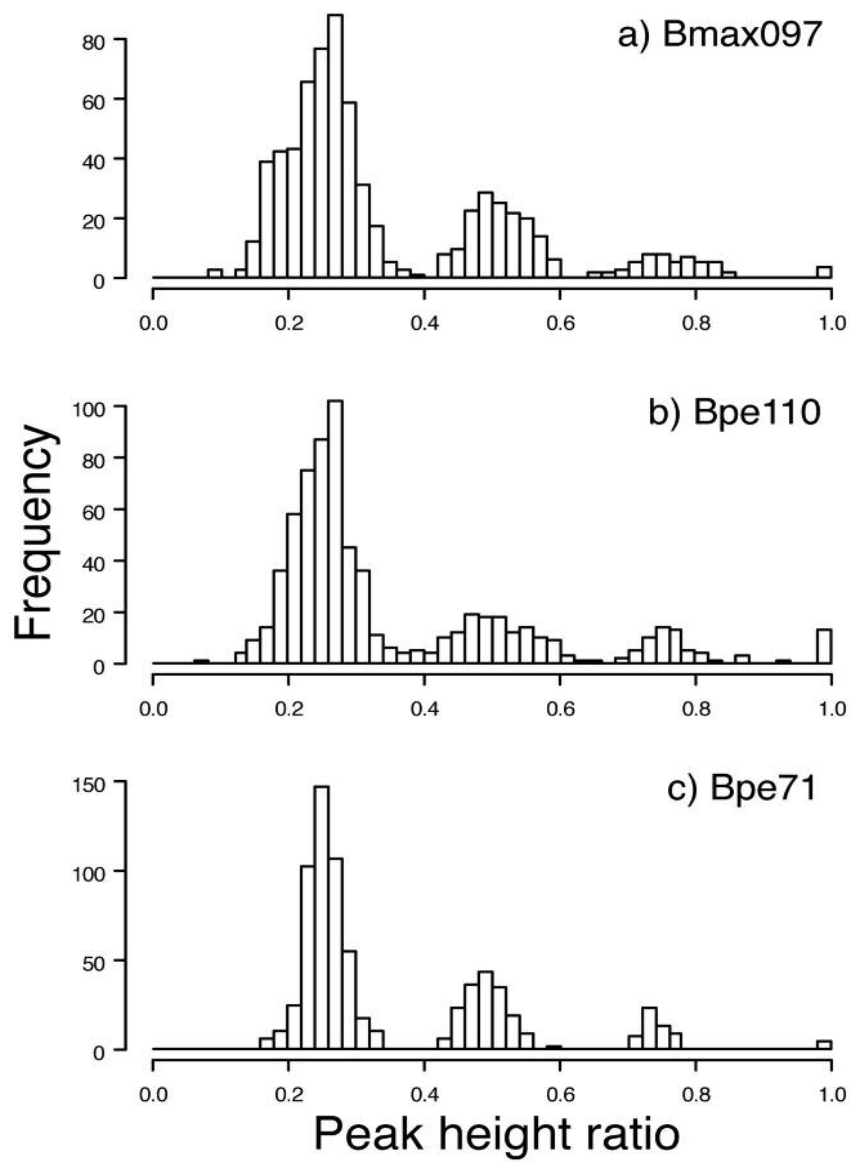

Figure 2. - Frequency distributions in the ratio of the corrected peak height of PCR products from alleles at three loci of nuclear microsatellites in each individual of Betula apoiensis. segregation pattern of the possible groupings in case (3). We verified the statistical differences between the observed and expected frequencies of the gamete genotypes using Chi-squared tests (chisq.test in R 3.0.2).

\section{Results}

We calculated the ratio of the corrected peak height for the three parents (A, B, and C) at the three loci (Bmax097, Bpe110, and Bpe71) as well as 240, 244, and 253 progeny individuals at Bmax097, Bpe110, and Bpe71, respectively. Frequency distributions of the peak height ratios at the three loci had four modes corresponding to the expected ratios, $0.25,0.5,0.75$, and 1 , which reflected the allele copy numbers, 1, 2, 3, and 4, respectively (Fig. 2). Among loci, these modes were the most clearly separated from one another at Bpe71 (Fig. 2c), which had the lowest stutter peaks (Table 1). We selected two thresholds separating three modes corresponding to the allele copy numbers, 1,2 , and 3 , at 0.40 and 0.62 for Bmax097 (Fig. 2a), at 0.38 and 0.64 for Bpe110 (Fig. 2b), and at 0.38 and 0.62 for Bpe71 (Fig. 2c). On the basis of these criteria, we inferred the tetraploid genotypes of the three parents at the three loci (Fig. 1) as well as 235, 241, and 252 progeny individuals at Bmax097, Bpe110, and Bpe71, respectively (Table 2). Thus, we could not infer genotypes of five, three, and one progeny individuals at Bmax097, Bpe110, and Bpe71, respectively, due to impossible allelic configurations: one $\mathrm{AAB}$ ( 0.5 and 0.25 ratios), two $\mathrm{ABC}(0.25$, 0.25 , and 0.25$)$, four $\mathrm{AAABC}(0.75,0.25$, and 0.25$)$, and two $\operatorname{AABCD}(0.5,0.25,0.25$, and 0.25).

We assigned diploid genotypes to gametes from the parents that created the progeny at Bmax097 and Bpe110 in the six reciprocal crosses (a1, a2, b1, b2, c1, and c2; Table 2). At Bmax097, we were not able to assign genotypes to eight gametes for three and one progeny individuals in crosses a 2 and b1, respectively, due to their unexpected configuration of alleles from both of their parents (Table 2). Genotypes of two of the 
Table 3. - The observed and expected frequencies of gamete genotypes from three parents of Betula apoiensis at three loci of nuclear microsatellites.

\begin{tabular}{llllll}
\hline Gamete & \multicolumn{4}{c}{ Frequency } \\
\cline { 2 - 5 } genotype & Observed & \multicolumn{4}{c}{ Expected } \\
\cline { 2 - 5 } & & Tet & DR & Di1 & Di2 \\
\hline
\end{tabular}

Locus Bmax097

Parent A

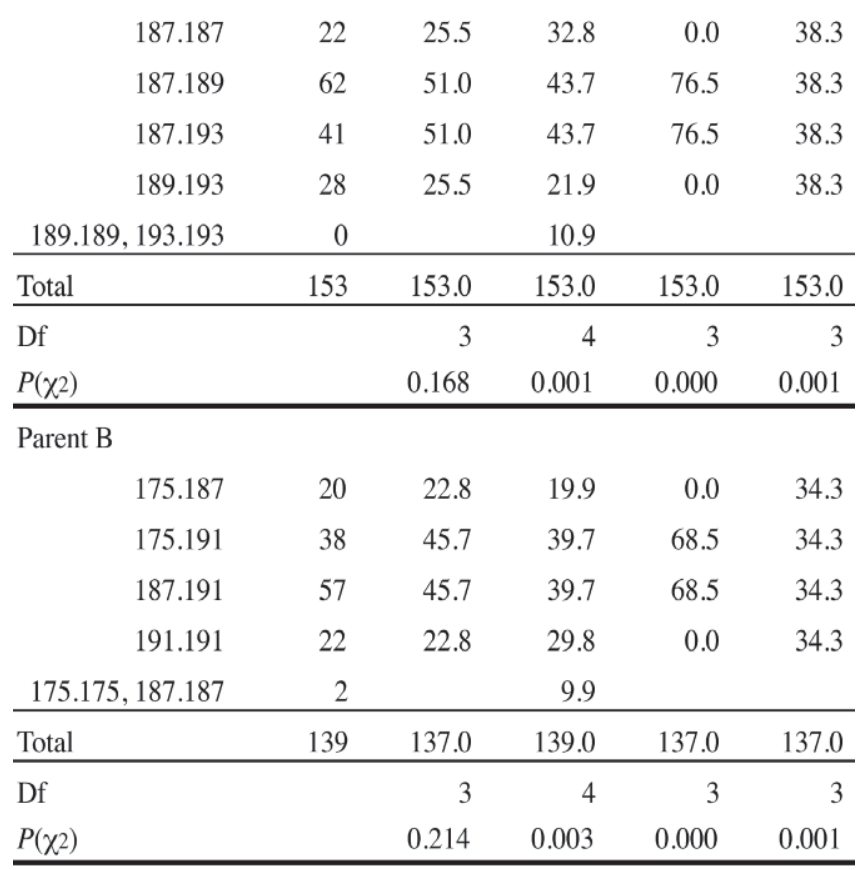

Parent C

\begin{tabular}{lrrrrr} 
& 191.191 & 95 & 85.0 & 91.1 & 85.0 \\
& 191.193 & 75 & 85.0 & 72.9 & 85.0 \\
& 193.193 & 0 & & 6.1 & \\
\hline Total & & 170 & 170.0 & 170.0 & 170.0 \\
\hline Df & & & 1 & 2 & 1 \\
$P\left(\chi^{2}\right)$ & & & 0.125 & 0.043 & 0.125 \\
\hline
\end{tabular}

\begin{tabular}{llllll}
\hline Gamete & \multicolumn{4}{c}{ Frequency } \\
\cline { 2 - 5 } genotype & Observed & \multicolumn{4}{c}{ Expected } \\
\cline { 2 - 5 } & & Tet & DR & Di1 & Di2 \\
\hline
\end{tabular}

Locus Bpe110

Parent A

\begin{tabular}{|c|c|c|c|c|c|c|}
\hline & 114.116 & 32 & 27.7 & 23.7 & 0.0 & 41.5 \\
\hline & 114.118 & 43 & 55.3 & 47.4 & 83.0 & 41.5 \\
\hline & 116.118 & 58 & 55.3 & 47.4 & 83.0 & 41.5 \\
\hline & 118.118 & 33 & 27.7 & 35.6 & 0.0 & 41.5 \\
\hline 114.11 & 116.116 & 0 & & 11.9 & & \\
\hline Total & & 166 & 166.0 & 166.0 & 166.0 & 166.0 \\
\hline Df & & & 3 & 4 & 3 & 3 \\
\hline$P\left(\chi_{2}\right)$ & & & 0.205 & 0.001 & 0.000 & 0.015 \\
\hline \multicolumn{7}{|c|}{ Parent B } \\
\hline & 112.112 & 77 & 75.5 & 80.9 & 75.5 & \\
\hline & 112.120 & 74 & 75.5 & 64.7 & 75.5 & \\
\hline & 120.120 & 0 & & 5.4 & & \\
\hline Total & & 151 & 151.0 & 151.0 & 151.0 & \\
\hline Df & & & 1 & 2 & 1 & \\
\hline$P\left(\chi^{2}\right)$ & & & 0.807 & 0.032 & 0.807 & \\
\hline \multicolumn{7}{|c|}{ Parent C } \\
\hline & 112.112 & 19 & 27.5 & 35.4 & 0.0 & 41.3 \\
\hline & 112.118 & 119 & 110.0 & 94.3 & 165.0 & 82.5 \\
\hline & 118.118 & 27 & 27.5 & 35.4 & 0.0 & 41.3 \\
\hline Total & & 165 & 165.0 & 165.0 & 165.0 & 165.0 \\
\hline Df & & & 2 & 2 & 2 & 2 \\
\hline$P\left(\chi_{2}\right)$ & & & 0.185 & 0.001 & 0.000 & 0.001 \\
\hline
\end{tabular}

four progeny individuals comprised only alleles from their mothers (Table 2). At Bpe71, we were not able to assign genotypes to gametes in crosses a1, a2, c1, and c2 because parent $\mathrm{A}$ shared more than two common alleles with the other parent in these crosses (Fig. 1). Thus, we assigned genotypes at Bpe71 to gametes for 42 and 45 progeny individuals in crosses $\mathrm{b} 1$ and $\mathrm{b} 2$, respectively (Table 2).

The observed frequencies of the gamete genotypes at Bmax097 and Bpe110 did not significantly differ among the four reciprocal crosses of each parent $(P=0.491$ in A, $P=0.051$ in $\mathrm{B}$, and $P=0.051$ in $\mathrm{C}$ at $\mathrm{Bmax} 097 ; P=0.334$ in $\mathrm{A}, P=0.311$ in $\mathrm{B}$, and $P=0.202$ in $\mathrm{C}$ at Bpe110; Table 2). At Bpe71, significant differences were not observed among the two crosses of each parent $(P=0.900$ in B and $P=0.147$ in C; Table 2). Therefore, the segregation patterns of the gamete genotypes seemed to be consistent between genders and between mates. Thus, we pooled

the observed frequencies of gamete genotypes from each parent at each locus among the four crosses (Table 3).

We assigned diploid genotypes to 462 and 482 gametes for 231 and 241 progeny individuals at Bmax097 and Bpe110, respectively, from the three parents and 174 gametes for 87 progeny individuals from parents B and $\mathrm{C}$ at Bpe71 (Tables 2, 3). Homozygous genotypes that seemed to be derived from double reduction occurred only in two gametes of parent B at Bmax097 (Table 3). Because the expected frequencies of homozygous genotypes from double reduction were low, we joined the frequencies of these homozygous genotypes in the following statistical tests (Table 3). The observed frequencies of the gamete genotypes from the three parents at the three loci did not significantly differ from the frequencies expected from tetrasomic inheritance without double reduction $(P>0.125$; Table 3$)$. On the other hand, the observed frequencies significantly differed from the frequencies expected from tetrasomic inheritance with double reduction at the maximum incidence $(P<0.043)$ 
Table 3. - (Continued).

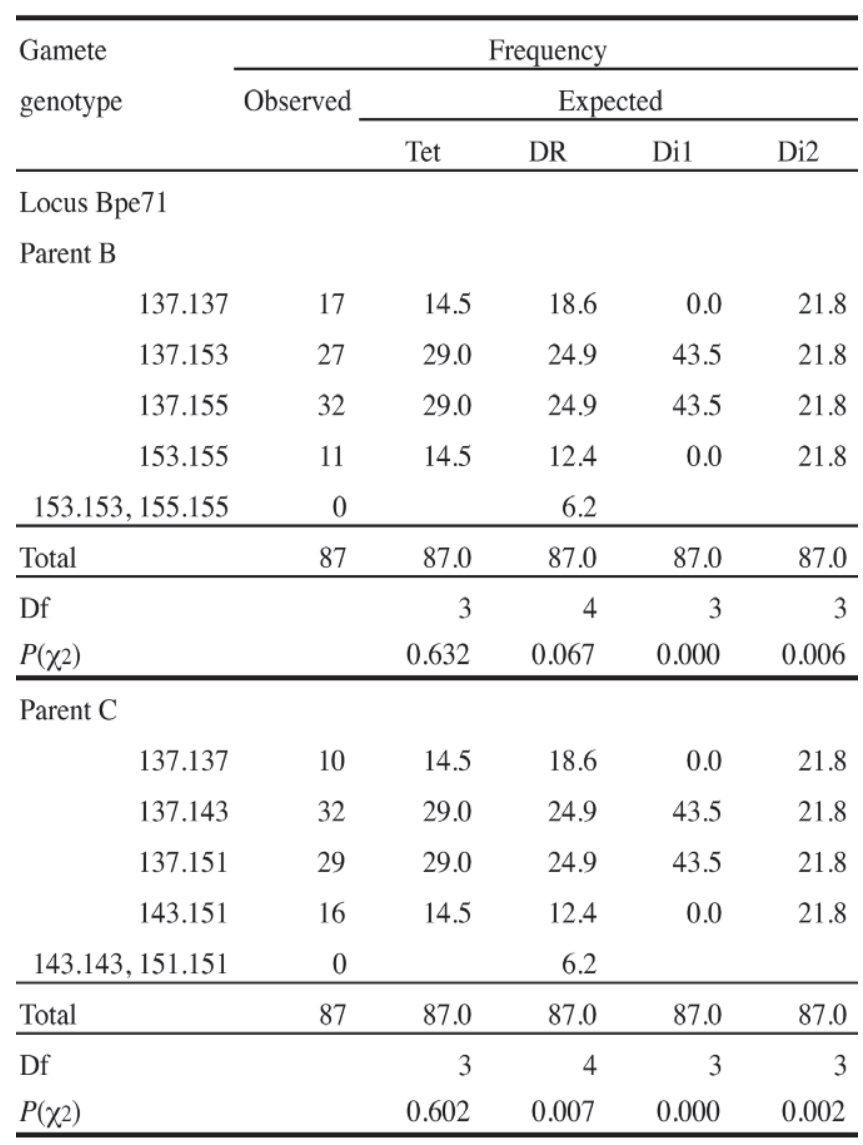

The frequencies are expected from tetrasomic inheritance without double reduction (Tet) and with double reduction at the maximum incidence (DR) and two segregation patterns in disomic inheritance (Di1 and Di2).

Df and $P\left(\chi^{2}\right)$ are the degree of freedom and the $P$ value, respectively, of a Chi-squared test to compare the observed frequencies and the positive frequencies expected from the modes of inheritance.

except for parent B at Bpe71 $(P=0.067$; Table 3$)$. In parent $C$ at Bmax097 and parent $B$ at Bpe110, segregation patterns of disomic inheritance were not discriminated from those of tetrasomic inheritance without double reduction (Table 3 ). In other cases, the observed frequencies significantly differed from the frequencies expected from disomic inheritance $(P<0.015$; Table 3$)$.

\section{Discussion}

\section{Inference of allelic dosages}

It is essential to infer allelic dosages in genetic studies on polyploids although precise assignment of polyploid genotypes had been difficult (DUFRESNE et al., 2014). Using the amount of PCR products, allelic configurations in nuclear microsatellites were inferred in polyploid plants (ESSELINK et al., 2004; NYBOM et al., 2004; LANDERGOTT et al., 2006; LUTTIKHUIZEN et al. 2007; Lo et al., 2009). In our method, we inferred tetraploid genotypes considering quantitatively both amplification bias- es and overlaps with stutter products in PCRs. Among the progeny, in which we measured peak heights of the PCR products, we successfully inferred the tetraploid genotypes of $97.9 \%(235 / 240)$ at Bmax097, 98.8\% $(241 / 244)$ at Bpe110, and $99.6 \%(252 / 253)$ at Bpe71. At Bpe71, the ratios of the corrected peak height were clearly discriminated among modes corresponding to the allele copy numbers (Fig. 2c), while the ratios were not so obviously separated at the other two loci, Bmax097 and Bpe110 (Fig. 2a,b). Thus, the inference of tetraploid genotypes in nuclear microsatellites using our method is not always successful and the success rate varies among loci.

Genotyping of nine progeny individuals were failed due to impossible allelic configurations. The summed ratios of corrected peak heights were less or more than 1 in these configurations. Null alleles can be responsible for three configurations, in which the summed ratios are less than 1, such as $\mathrm{AAB}$ ( 0.5 and 0.25 ratios), two $\mathrm{ABC}$ $(0.25,0.25$, and 0.25$)$. Null alleles are relatively frequent in polyploids because of the failure of amplification in homoeologous loci in allopolyploids, the loss of primer binding sites due to transposon activity and genomic rearrangement, and the increased bias of amplification among alleles (DUFRESNE et al., 2014). Among the examined progeny, $0.4 \%(3 / 727)$ individuals may have null alleles, which are too few to assume that null alleles are inherited from parents to progeny. Thus, null alleles seem to be absent in our family samples, and artifacts in PCRs may cause the impossible allelic configurations. Family samples are useful to detect and exclude null alleles in tetraploids, in particulr using parents with four different alleles.

Because the parents shared a limited number of common alleles owing to high polymorphism in microsatellites, we assigned diploid genotypes to the gametes on the basis of the tetraploid genotypes of the parents and their progeny in most of the crosses (Fig. 1). At Bmax097, four progeny genotypes could not derive from their both parents, and two of the four unexpected genotypes comprised only alleles from their mothers. This result may suggest apomixis or selfing, which has been reported in birches (ERIKKSON and JONSSON, 1986), due to contamination of the self pollen in the cross treatment. We conducted a pollination experiment with only-bagging, selfing, and outcrossing treatments and a natural condition in $B$. apoiensis (NAGAMITSU et al., 2006a). This experiment demonstrated different proportions in seed set and seed germination: the outcrossing treatment $(0.42-0.63)>$ the natural condition $(0.08-0.11)>$ the selfing treatment $(0.02-0.06)>$ the only-bagging treatment $(0.00-0.01)$. This result indicates that apomixis is almost absent, and that selfing is rare. Thus, the possibility of apomixis or selfing seems to be low, and artifacts in PCRs may cause the unexpected genotypes.

\section{Inference of inheritance modes}

Differences in segregation patterns of gamete genotypes were not evident in the progeny arrays. Thus, the observed frequency of the gamete genotypes indicates a consistent mode of allelic segregation at meiosis. Disom- 
ic inheritance was rejected in all parents and loci $(6 / 6$ cases), but tetrasomic inheritance was not refused in any parents and loci (0/8 cases), without regard to double reduction (Table 3 ). However, because only three loci were examined, it is necessary to confirm the mode of inheritance at more loci. In polyploids, inheritance patterns can vary among individuals within populations and among loci within individuals (DUFRESNE et al., 2014). In polyploid birches, quadrivalents were often observed at low frequencies, suggesting the affinity between pairs of bivalents and the homoeology among polyploid chromosomes (BROWN and AL-DAwOODY, 1979). Hybrids between two tetraploid species, B. pubescens and B. cordifolia, showed a disomic pattern (STERN, 1965). However, one of the two species, B. pubescens, which seemed to have an allopolyploid origin (BROwN and AL-DAwOODY, 1979; JÄRVINEN et al., 2004), exhibited a tetrasomic pattern (STERN, 1965). According to a phylogenetic reconstruction of several birch species, two putative progenitor species of $B$. apoiensis, B. ermanii Cham. and B. ovalifolia Rupr., are not closely related (NAGAMITSU et al., 2006b). These findings suggest that homoeologous chromosomes originating from different progenitor species, even if they are not closely related, are able to pair with each other in poplyploid birches.

In tetrasomic inheritance, double reduction at a certain locus potentially occurs due to crossovers between the centromere and the locus (BEVER and FELBER, 1992). In $B$. apoiensis, double reduction seemed to be rare because tetrasomic segregation patterns expected from the maximum incidence of double reduction were rejected in most parents and loci (7/8 cases; Table 3$)$. In birches, the shape of chromosomes is almost spherical, and their size is relatively small (BROWN and AL-DAwOODY, 1979), ranging from 0.6 to $1.8 \mu \mathrm{m}$ (TAPER and GRANT, 1973). These features of chromosomes in birches seem to be related to a low frequency of crossovers, which may result in the rare double reduction.

\section{Implications for future studies}

Our method to infer tetraploid genotypes in nuclear microsatellites seems to be successful in family samples with few null alleles when amplification biases and stutter-product overlaps are accessed properly. Because the accuracy of inference of allelic dosages varied among loci, genotyping is failed at part of loci in nuclear microsatellites. Thus, utilization of a large number of loci, which have been identified and are available in polyploid birches (TRUONG et al., 2005), can hedge the risk of genotyping failure.

Other approaches to study tetraploids to use microsatellites inherited in a diploid fashion and to use diploid genotypes of gametes. Specific primers that amplify only in the diploid component in allopolyploids are useful in the former approach (KoRBECKA et al., 2011). A technique for genotyping of a single pollen grain is available for the latter approach (MATSUKI et al., 2007). Microsatellite are effective markers in population genetics. Thus, their application to polyploids is challenging, and will be fruitful, when the difficulties in inference of allelic dosages and inheritance modes are overcome.

\section{Acknowledgements}

We would like to thank the Agency for Cultural Affairs and the government of Hokkaido for permission to conduct this study, the Education Committee of Samani Town and the Mt. Apoi Fun Club for being so kind as to make arrangements for the field work, and MAYUKO HotTa, Yosuke Mori, Yukiko SAKAMoto, Makiko Sumita, and RIKA YoshidA for their assistance with the field and laboratory work. This study was supported by a grant for national institutes involved in pollution prevention and natural environmental conservation from the Ministry of the Environment of Japan.

\section{References}

Bever, J. D. and F. FELBER (1992): The theoretical population genetics of autopolyploidy, pp. 185-217. In: Oxford Surveys in Evolutionary Biology, edited by J. Antonovics and D. Futuyma, Oxford University Press, New York.

Brown, I. R. and D. AL-Dawoody (1979): Observations on meiosis in three cytotypes of Betula alba L. New Phytologist 83: 801-811.

DAKIN, E. E. and J. C. Avise (2004): Microsatellite null alleles in parentage analysis. Heredity 93: 504-5099.

De Silva, H. N., A. J. Hall, E. Rikkerink, M. A. McNeIlage and L. G. Fraser (2005): Estimation of allele frequencies in polyploids under certain patterns of inheritance. Heredity 95: 327-334.

Dufresne, F., M. Stift, R. Vergilino and B. K. Mable (2014): Recent progress and challenges in population genetics of polyploid organisms: an overview of current state-of-the-art molecular and statistical tools. Molecular Ecology 23: 40-69.

ErikKson, G. and A. Jonsson (1986): A review of the genetics of Betula. Scandinavian Journal of Forest Research 1: 421-434.

Esselink, G. D., H. Nybom and B. Vosman (2004): Assignment of allelic configuration in polyploids using the MAC-PR (microsatellite DNA allele counting-peak ratios) method. Theoretical and Applied Genetics 109: 402-408.

FurLow, J. J. (1990): The genera of Betulaceae in the southeastern United States. Journal of Arnold Arboretum 71: 1-67.

Järvinen, P., A. Palmé, L. O. Morales, M. LännenpäÄ, M. Keinänen, T. Sopanen and M. LAscoux (2004): Phylogenetic relationships of Betula species (Betulaceae) based on nuclear $\mathrm{ADH}$ and chloroplast matK sequences. American Journal of Botany 91: 1834-1845.

Korbecka, G., A. Hamilton and J. R. Pannell (2011): Mixed mating in androdioecious Mercurialis annua inferred using progeny arrays and diploid-acting microsatellite loci in a hexaploid background. Annals of Botany 107: 1057-1061.

KulJu, K. K. M., M. PekKinen and S. VARvio (2004): Twenty-three microsatellite primer pairs for Betula pendula (Betulaceae). Molecular Ecolology Notes 4: 471-473.

LANDERgotT, U., Y. NACIRI, J. J. Schneller and R. HOLDEREGGER (2006): Allelic configuration and polysomic inheritance of highly variable microsatellites in tetraploid gynodioecious Thymus praecox agg. Theoretical and Applied Genetics 113: 453-465. 
Leitch, I. J. and M. D. Bennett (1997): Polyploidy in angiosperms. Trends in Plant Science 2: 470-476.

LI, J., S. Shoup and Z. CHEN (2005): Phylogenetics of Betula (Betulaceae) inferred from sequences of nuclear ribosomal DNA. Rhodora 107: 69-86.

Lo, E. Y. Y., S. Stefanović and T. Dickinson (2009): Population genetic structure of diploid sexual and polyploid apomictic hawthorns (Crataegus; Rosaceae) in the Pacific Northwest. Molecular Ecology 18: 1145-1160.

LutTikhuizen, P. C., M. Stift, P. Kuperus and P. H. van Tienderen (2007): Genetic diversity in diploid vs. tetraploid Rorippa amphibia (Brassicaceae). Molecular Ecology 16: 3544-3553.

MAtHeR, K. (1936): Segregation and linkage in autotetraploids. Journal of Genetics 32: 287-314.

Matsuki, Y., Y. IsAGI and Y. SuYAMA (2007): The determination of multiple microsatellite genotypes and DNA sequences from a single pollen grain. Molecular Ecology Notes 7: 194-198.

Nagamitsu, T., T. KaWAhara and A. Kanazashi (2006a): Pollen-limited production of viable seeds in an endemic dwarf birch Betula apoiensis and incomplete reproductive barriers to a sympatric congener B. ermanii. Biological Conservation 129: 91-99.

NAGAmitsu, T., T. KaWAHARA and A. KANAZASHI (2006b): Endemic dwarf birch, Betula apoiensis (Betulaceae), is a hybrid that originated from $B$. ermanii and B. ovalifolia. Plant Species Biology 21: 19-29.

Nybom, H., G. D. Esselink, G. Werlemark and B. Vosman (2004): Microsatellite DNA marker inheritance indicates preferential pairing between two highly homologous genomes in polyploid and hemisexual dog-roses, Rosa L. Sect. Caninae DC. Heredity 92: 139-150.

Ogyu, K., Y. Tsuda, T. Sugaya, H. Yoshimaru and Y. Ide (2003): Identification and characterization of microsatellite loci in Betula maximowicziana Regel. Molecular Ecology Notes 3: 268-269.

OLson, M. S. (1997): Bayesian procedures for discriminating among hypotheses with discrete distributions: Inheritance in the tetraploid Astilbe biternata. Genetics 147: 1933-1942.
Palme, A. E., Q. Su, S. Palsson and M. Lascoux (2004): Extensive sharing of chloroplast haplotypes among European birches indicates hybridization among Betula pendula, B. pubescens and B. nana. Molecular Ecology 13: $167-178$.

R Development Core Team (2013): R: a language and environment for statistical computing. R Foundation for Statistical Computing, Vienna, http://www.R-project.org

Ronfort, J., E. JENCZEWski, T. BATAILlon and F. RousseT (1998): Analysis of population structure in autotetraploid species. Genetics 150: 921-930.

Schenk, M. F., C. N. Thienpont, W. J. M. Koopman, L. J. W. J. Gilissen and M. J. M. SMulders (2008): Phylogenetic relationships in Betula (Betulaceae) based on AFLP markers. Tree Genetics and Genomes 4: 911-924.

Schumer, M., G. G. Rosenthal and P. Andolfatto (2014): How common is homoploid hybrid speciation? Evolution 68: $1553-1560$.

Soltis, D. E., P. S. Soltis and J. A. TAte (2003): Advances in the study of polyploidy since plant speciation. New Phytologist 161: 173-191.

Soltis, P. S. and D. E. Soltis (2000): The role of genetic and genomic attributes in the success of polyploids. Proceedings of the National Academy of Science of the USA 97: 7051-7057.

SteRn, K. (1965): Tetrasome spaltung bei Betula pubescens. Silvae Genetica 14: 56-57.

TAPER, L. J. and W. E. GRANT (1973): The relationship between chromosome size and DNA content in birch (Betula) species. Caryologia 26: 263.

Thórsson, A. T., E. SAlmela and K. AnamthaWAT-Jónsson (2001): Morphological, cytogenetic, and molecular evidence for introgressive hybridization in birch. Journal of Heredity 92: 404-408.

Truong, C., A. E. Palme, F. Felber and Y. Naciri-Graven (2005): Isolation and characterization of microsatellite markers in the tetraploid birch, Betula pubescens ssp. tortuosa. Molecular Ecology Notes 5: 96-98. 